Pacific Journal of Mathematics

NON-LINEAR DIFFERENTIAL EQUATIONS ON CONES IN 


\title{
NON-LINEAR DIFFERENTIAL EQUATIONS ON CONES IN BANACH SPACES
}

\author{
Charles V. Coffman
}

1. Introduction. Let $y$ denote a vector in $n$-dimensional Euclidean space $E^{n}, y \geqq 0$ means that each component of $y$ is nonnegative. Let $f(t, y)$ be a function with values in $E^{n}$ defined on the domain $D=$ $\left\{(t, y), 0 \leqq t<\infty, y \in E^{n}, y \geqq 0\right\}$. Assume that $f(t, y)$ is continuous on $D$ and that for each $(t, y) \in D,-f(t, y) \geqq 0$, and that $f(t, 0) \equiv 0$. Let $C$ be a positive constant. It is known, [*,4], that the differential equation $d y / d t=f(t, y)$ possesses at least one solution $y=y(t)$, defined for $0 \leqq t<\infty$, with $y(t) \geqq 0$ for $0 \leqq t<\infty$, and satisfying $|y(0)|=C$, where $|\cdots|$ denotes the Euclidean norm in $E^{n}$. (Actually the hypothesis of the theorem referred to involves an additional condition on $f(t, y)$, however the proof given in [4] can be modified so as to avoid the need for this condition.)

It was shown in [2] that the linear case of the result just quoted has a generalization to an infinite dimensional case. Before describing this result it will be necessary to introduce the following terminology and notation. Throughout the remainder of the paper $Y$ will denote a (real) Banach space with norm $\|\cdots\|$ and $K$ some fixed, closed convex cone in $Y$. For $y \in Y, y \geqq 0$ will mean $y \in K$. A linear operator $A$ on $Y$ will be said to be nonnegative if $A y \geqq 0$ whenever $y \geqq 0$. A nonempty set of the form $H=H\left(y^{*}\right)=\left\{y: y \in K, y^{*}(y)=1\right\}$, where $y^{*}$ is in the dual space $Y^{*}$ of $Y$ will be called a cross-section of the cone $K$. Theorem 1 of [2] concerns the linear differential equation on the Banach space $Y$,

$$
d y / d t=-A(t) y,
$$

where for each $t, 0 \leqq t<\infty, A(t)$ is a nonnegative bounded linear operator on $Y$, and $A(t)$ is strongly continuous on $0 \leqq t<\infty$. The result states that if the cone $K$ has a weakly compact cross-section then the differential equation (1.1) has a nontrivial solution $y=y(t)$ satisfying $y(t) \geqq 0$ for $0 \leqq t<\infty$.

Theorem 3 of [2] is the dual of Theorem 1 and concerns the differential equation on $Y^{*}$ adjoint to (1.1)

$$
d y^{*} / d t=-A^{*}(t) y^{*}
$$

where for each $t, 0 \leqq t<\infty, A^{*}(t)$ is the adjoint of $A(t)$. Let $A(t)$ be as above, this theorem states that if $K$ has an interior point then (1.2) has a nontrivial solution $y^{*}=y^{*}(t)$ satisfying $y^{*}(t) \in K^{*}$ for $0 \leqq t<\infty$. $K^{*}$ in $Y^{*}$ is the dual cone of the cone $K$ in $Y$, consisting of those

Received May 13, 1963. 
elements $y^{*}$ such that $y^{*}(y) \geqq 0$ for each $y \in K$.

The main result of this note, Theorem 4.1, generalizes the result of [4] mentioned above to an infinite dimensional case and contains both of the results just quoted.

The following standard terminology will be used. A closed linear manifold $\Gamma$ in the dual space $Y^{*}$ of $Y$ is called determining for $Y$ if, when $y \in Y, \gamma(y)=0$ for each $\gamma \in \Gamma$ implies $y=0$. Such a manifold defines a topology, referred to as the weak $\Gamma$-topology, on $Y$. The generalized sequence $\left\{y_{\alpha}\right\}$ of elements of $Y$ has limit 0 in the weak $\Gamma$ topology if and only if $\lim _{\alpha} \gamma\left(y_{\alpha}\right)=0$ for each $\gamma \in \Gamma$.

2. Differential equations on a Banach space. Let $\Gamma$ be a closed linear manifold in $Y^{*}$ which is determining for $Y$. A function $y(t)$ on some interval $I$ on the real line, with values in $Y$, will be said to have a weak $\Gamma$-derivative, or to be weakly $\Gamma$-differentiable at $t \in I$ if the limit as $h \rightarrow 0$ of the difference quotient $(y(t+h)-y(t)) / h$ exists in the weak $\Gamma$-topology. If $y(t)$ is weakly $\Gamma$-differentiable on $I$, its weak $\Gamma$-derivative will be denoted by $D_{F} y(t)$. Let $C$ be a subset of $Y$ and let $I$ be, as above, an interval on the real line. Consider the differential equation

$$
d y / d t=f(t, y)
$$

where $f(t, y)$ is a function from $I \times C \rightarrow Y$, continuous in the weak $\Gamma$-topology. Let $y(t)$ be a function on $I$ with values in $C$. If $y(t)$ is strongly continuous and strongly differentiable on $I$ with a strongly continuous derivative $d y / d t$, and if (2.1) holds, $y(t)$ will be said to be a strong solution of (2.1) on $I$. If $y(t)$ is strongly continuous, weakly absolutely continuous and strongly differentiable a.e. on $I$, and if (2.1) holds a.e. on $I, y(t)$ will be said to be a strong solution in the extended sense of (2.1) on $I$. Finally $y(t)$ will be said to be a weak $\Gamma$-solution of (2.1) on $I$ if it is weakly $\Gamma$-differentiable on $I$ and if $D_{I} y(t)=f(t, y(t))$ on $I$. Obviously if $y(t)$ is a weak $\Gamma$-solution of (2.1) then $D_{\Gamma} y(t)$ is weakly $\Gamma$-continuous.

LEMMA 2.1. If $y(t)$ is a strong solution in the extended sense of (2.1) on I, then it can be expressed as the Bochner integral of its derivative.

Proof. Since $y(t)$ is strongly continuous, its range lies in a separable subspace of $Y$. Clearly the values of $d y / d t$, where it exists will be in the same subspace. Put

$$
u(t)=f(t, y(t))
$$


then $u(t)$ is weakly $\Gamma$-continuous and coincides with $d y / d t$ a.e. where the latter is defined. Since $u(t)$ is almost separably valued and weakly $\Gamma$-continuous, it follows from Theorem 1.1.7, [3, p. 330], that $u(t)$, hence $d y / d t$ is locally Bochner integrable on $I$. Since $y(t)$ is weakly absolutely continuous it may be expressed as the indefinite integral of $d y / d t$, Theorem 3.8.6, [5].

Lemma 2.2. Let $y(t)$ be a function defined on I with values in $C$. In order that $y(t)$ be a strong solution in the extended sense of (2.1), it is necessary and sufficient that $y(t)$ be a weak $\Gamma$-solution of (2.1) and that $D_{\Gamma} y(t)$ be almost separably valued.

REMARK. If $\Gamma=Y^{*}$ and if $y(t)$ is a weak $\Gamma$-solution of (2.1), then $D_{\Gamma} y(t)$ is necessarily separably valued, since it is weakly $\Gamma$-continuous; see [5], p. 59.

Proof of Lemma 2.2. Necessity. Let $y(t)$ be a strong solution in the extended sense of (2.1). Let $u(t)$ be defined by (2.2). From the proof of Lemma 2.1, $u(t)$ is almost separably valued and for $t, t^{\prime} \in I$, $y(t)-y\left(t^{\prime}\right)=\int_{t^{\prime}}^{t} u(t) d t$. For each $\gamma \in \Gamma, \gamma(u(t))$ is continuous, consequently $\gamma(y(t)) \in C^{1}$ and $d[\gamma(y(t))] / d t=\gamma(u(t))$, since one has $\gamma\left[\int_{t^{\prime}}^{t} u(t) d t\right]=$ $\int_{t^{\prime}}^{t} \gamma(u(t)) t$. It follows that $D_{\Gamma} y(t)$ exists everywhere on $I$ and that $\vec{D}_{\Gamma} y(t)=u(t)$.

Sufficiency. Let $y(t)$ be a weak $\Gamma$-solution of (2.1) and let $D_{\Gamma} y(t)$ be almost separably valued. By Theorem 1.1.7, [3], $D_{\Gamma} y(t)$ is locally Bochner integrable on $I$. For each $\gamma \in \Gamma, \gamma\left(D_{\Gamma} y(t)\right)$ is continuous on $I$, so for $t, t^{\prime} \in I, \gamma(y(t))-\gamma\left(y\left(t^{\prime}\right)\right)=\int_{t^{\prime}}^{t} \gamma\left(D_{\Gamma}^{\urcorner} y(t)\right) d t=\gamma\left[\int_{t^{\prime}}^{t} D_{\Gamma} y(t) d t\right]$. Since $\Gamma$ is determining for $Y$ it follows that $y(t)$ can be expressed as the indefinite Bochner integral of $D_{\Gamma} y(t)$. Hence $y(t)$ is in fact strongly absolutely continuous, and, by Corollary 2, p. 88, [5], $y(t)$ is strongly differentiable a.e., and $d y / d t$ coincides a.e., where it is defined with $D_{\Gamma} y(t)$.

3. Convergence of approximate solutions. Let $Y, \Gamma, C, I$, and $f(t, y)$ be as in $\S 2$. The following generalization of the notion of an $\varepsilon$-approximate solution of a differential equation will be used. Let $V$ be a neighborhood of 0 in the weak $\Gamma$-topology. A function $y(t)$ will be called a $V$-approximate weak $\Gamma$-solution of (2.1) on $I$ if

(i) $y(t)$ is defined on $I$, has values in $C$ and is weakly $\Gamma$-continuous;

(ii) for some finite set of points $S$ on $I, D_{\Gamma} y(t)$ exists and is weakly $\Gamma$-continuous on $I-S$, further $D_{\Gamma} y(t)$ has only simple discontinuities, in the weak $\Gamma$-topology, at points of $S$;

(iii) $\left(D_{\Gamma} y(t)-f(t, y)\right) \in V$ for $t \in I-S$. 
REMARK. In view of the principal of uniform boundedness (ii) implies that $\left\|D_{\Gamma} y(t)\right\|$ is locally bounded on $I$.

LEMmA 3.1. Let $\left\{y_{n}(t)\right\}$ be a sequence of weakly $\Gamma$-continuous functions defined on $I$ with values in $C$. Let $C$ be compact in the weak $\Gamma$-topology. For each neighborhood $V$ of 0 , in the weak $\Gamma$-topology, and for each compact subinterval $I^{\prime}$ of $I$, let there exist an $N=N\left(V, I^{\prime}\right)$ such that for all $n \geqq N, y_{n}(t)$ is a $V$-approximate weak $\Gamma$-solution of (2.1) on $I^{\prime}$. Then there exists a subsequence $\left\{y_{n_{k}}(t)\right\}$ which converges uniformly on every compact subinterval of $I$ to a weak $\Gamma$-solution $y(t)$ of (2.1) on $I$.

Proof. The existence of a subsequence convergent on each compact subinterval of $I$ will follow from Ascoli's theorem, [1, p. 43], if it is shown that the sequence is equicontinuous in the weak $\Gamma$-topology. Let $t_{0} \in I$ be arbitrary, it will suffice to show that for each $\gamma \in \Gamma$, and for each $\varepsilon>0$ there exists a $\delta=\delta\left(\varepsilon, \gamma, t_{0}\right)$ such that for $n=1,2, \cdots$

$$
\left|\gamma\left(y_{n}(t)-y_{n}\left(t_{0}\right)\right)\right|<\varepsilon
$$

whenever $t \in I$, and $\left|t-t_{0}\right|<\delta$. For some $\delta_{0}>0$, there exists, by the principle of uniform boundedness, an $M$ such that $\|f(t, y)\| \leqq M$ for $t \in I,\left|t-t_{0}\right| \leqq \delta_{0}$ and $y \in C$. For $n=1,2, \cdots$, one has

$$
\begin{array}{r}
\gamma\left(y_{n}(t)\right)-\gamma\left(y_{n}\left(t_{0}\right)\right)=\int_{t_{0}}^{t} \gamma\left(f\left(t, y_{n}(t)\right)\right) d t \\
+\int_{t_{0}}^{t} \gamma\left(D_{\Gamma} y_{n}(t)-f\left(t, y_{n}(t)\right)\right) d t .
\end{array}
$$

For all sufficiently large $n$, say $n \geqq n_{0},\left|\gamma\left(D_{\Gamma} y_{n}(t)-f\left(t, y_{n}(t)\right)\right)\right|<\varepsilon / 2\|\gamma\| \delta_{0}$, for $t \in I,\left|t-t_{0}\right|<\delta_{0}$. Thus, if $\delta_{1}<\min \left(\delta_{0}, \varepsilon / 2 M\|\gamma\| \delta_{0}\right)$ then (3.1) holds for $t \in I,\left|t-t_{0}\right|<\delta_{1}$ and $n \geqq n_{0}$. Clearly it is possible to choose a $\delta, 0<\delta \leqq \delta_{1}$, such that (3.1) holds for all $n$ when $t \in I,\left|t-t_{0}\right|<\delta$.

Let the subsequence $\left\{y_{n_{k}}(t)\right\}$ of the original sequence be uniformly convergent on each compact subinterval of $I$ to $y(t)$. For each $\gamma \in \Gamma$, $\gamma\left(f\left(t, y_{n_{k}}(t)\right) \rightarrow \gamma\left(f(t, y(t))\right.\right.$ and $\gamma\left(D_{\Gamma} y_{n_{k}}(t)-f\left(t, y_{n_{k}}(t)\right)\right) \rightarrow 0$ uniformly on each compact subinterval of $I$. It follows that $\gamma\left(y(t)-y\left(t_{0}\right)\right)=$ $\int_{t_{0}}^{t} \gamma(f(t, y(t))) d t$, for $t, t_{0} \in I$, for each $\gamma \in \Gamma$. Consequently $D_{\Gamma} y(t)$ exists on $I$ and $D_{\Gamma} y(t)=f(t, y(t))$ there.

4. An existence theorem. As a consequence of the results of the last section, one has the following local existence theorem for the problem (2.1).

THEOREM 4.1. Let the unit sphere in $Y$ be compact in the weak 
$\Gamma$-topology. For some real interval $I$, some point $y_{0} \in Y$ and some positive constant $k$ let $f(t, y)$ be defined and weakly $\Gamma$-continuous for $t \in I,\left\|y-y_{0}\right\| \leqq k$. Let $t_{0}$ be in the interior of $I$, then on some open subinterval $I^{\prime}$ of $I$, with $t_{0} \in I^{\prime}$, there exists a weak $\Gamma$-solution $y(t)$ of (2.1) satisfying the initial condition

$$
y\left(t_{0}\right)=y_{0} \text {. }
$$

Proof. It can be assumed that $I$ is compact, so that $\|f(t, y)\| \leqq$ $M<\infty$ for $t \in I,\left\|y-y_{0}\right\| \leqq k$. Let $\delta$ be some number on the range $0<\delta<k / M$, such that the interval $I^{\prime}=\left\{t:\left|t-t_{0}\right|<\delta\right\} \subset I$. For a positive integer $n$, subdivide $I^{\prime}$ by the points $t_{l}=t_{0}+l \delta / n, l=0$, $\pm 1, \cdots, \pm n-1$. Define a function $y_{n}(t)$ on $I^{\prime}$ as follows: let $y_{n}\left(t_{0}\right)=$ $y_{0}$, and let $y_{n}(t)=y_{0}+\left(t-t_{0}\right) f\left(t_{0}, y_{0}\right)$ for $t_{0} \leqq t \leqq t_{1}$, clearly then $\left\|y_{n}(t)-y_{0}\right\| \leqq M\left(t_{1}-t_{0}\right) \leqq k / n$ for $t_{0} \leqq t \leqq t_{1}$. Assume that $y_{n}(t)$ is defined for $t_{0} \leqq t \leqq t_{l}, l \leqq n-1$, with $\left\|y_{n}(t)-y_{0}\right\| \leqq l k / n$ for $t_{0} \leqq t \leqq$ $t_{l}$, and put $y_{n}(t)=y_{n}\left(t_{l}\right)+\left(t-t_{l}\right) f\left(t_{l}, y_{n}\left(t_{l}\right)\right)$ for $t_{l} \leqq t \leqq t_{l+1}$. Thus $\left\|y_{n}(t)-y_{0}\right\| \leqq(l+1) k / n$, and the process can be continued to define $y_{n}(t)$ on $t_{0} \leqq t<\delta$, with $\left\|y_{n}(t)-y_{0}\right\|<k$ there. $y_{n}(t)$ is defined to the left of $t_{0}$ in a similar fashion.

Let $y_{n}(t)$ be so defined for $n=1,2, \cdots$. The interval $I$ being compact, $f(t, y)$ is uniformly weakly $\Gamma$-continuous for $t \in I,\left\|y-y_{0}\right\| \leqq k$. It readily follows that, given $V \subset Y$, a neighborhood of zero in the weak $\Gamma$-topology, then for all sufficiently large $n, y_{n}(t)$ is a $V$-approximate weak $\Gamma$-solution of (2.1) on $I^{\prime}$.

The existence of a weak $\Gamma$-solution of (2.1) on $I^{\prime}$ satisfying the initial condition (4.1) now follows from Lemma 3.1.

5. Differential equations on cones. The main result of this note is the following.

THEOREM 5.1. Let $K$ be a closed convex cone in the Banach space $Y$. Let $\Gamma$ be as above, and let there exist $a \gamma_{0} \in K^{*} \cap \Gamma$ such that the cross-section $H\left(\gamma_{0}\right)$ of $K$ is compact in the weak $\Gamma$-topology on $Y$. Let $I=[0, \infty)$ and let $f(t, y)$ be defined on $I \times K$, and continuous in the weak $\Gamma$-topology, and suppose that

$$
-f(t, y) \in K \quad \text { for }(t, y) \in K .
$$

assume also that

$$
f(t, 0) \equiv 0 \text {. }
$$

Then there exists a weak $\Gamma$-solution $y(t)$ of (2.1) on $I$ with $y(t) \in K$ for all $t \in I$, and with $y(0) \in H$. 
Proof. First it will be shown that for each $T>0$ there exists a weak $\Gamma$-solution $y_{T}(t)$ of $(2.1)$ on $[0, T]$ with $y_{T}(t) \in K$ for $0 \leqq t \leqq T$, and having $y_{T}(0) \in H$. Let $T>0$ be given, let $n$ be a positive integer and let $\eta$ be an arbitrary element of $K$. Subdivide $[0, T]$ into $n$ subintervals of equal length, and construct a polygonal approximate solution $y_{n}(t)=y_{n}(t, \eta)$ as in $\S 4$, beginning at $t=T$ with $y_{n}(T, \eta)=$ $\eta$ and proceeding to the left. That is, $t_{k}=k T / n, k=0, \cdots, n$, being the endpoints of the intervals of the subdivision, if $y_{n}(t, \eta)$ is assumed to be defined on $\left[t_{k}, T\right], k>0$, the definition is extended to $\left[t_{k-1}, T\right]$ by putting

$$
y_{n}(t, \eta)=y_{n}\left(t_{k}, \eta\right)+\left(t-t_{k}\right) f\left(t_{k}, y_{n}\left(t_{k}, \eta\right)\right)
$$

on $\left[t_{k-1}, t_{k}\right]$. Notice that because of (5.1) at no point can the polygon leave $K$.

From the fact that $f(t, y)$ is weakly $\Gamma$-continuous it readily follows, using (5.3) and an induction argument that the mapping of $K \rightarrow K$ given by $\eta \rightarrow y_{n}(0, \eta)$ is continuous in the weak $\Gamma$-topology. From (5.2), it follows that $y_{n}(0,0)=0$. Because of $(5.1)$ one has, in view of (5.3) that $y_{n}(t, \eta) \geqq y_{n}\left(t^{\prime}, \eta\right)$ for $0 \leqq t<t^{\prime} \leqq T$, and consequently that

$$
\gamma_{0}\left(y_{n}(t, \eta)\right) \geqq \gamma_{0}\left(y_{n}\left(t^{\prime}, \eta\right)\right) \text { for } 0 \leqq t<t^{\prime} \leqq T .
$$

The weak $\Gamma$-continuity of the mapping $\eta \rightarrow y_{n}(0, \eta)$ implies therefore, the existence of values $\eta \in K$ for which $y_{n}(0, \eta) \in H$. For each $n=$ $1,2, \cdots$, let $\eta_{n}$ be chosen so that $y_{n}\left(0, \eta_{n}\right) \in H$. By (5.4) one has that for $n=1,2, \cdots y_{n}\left(t, \eta_{n}\right) \in K_{1}$ for $0 \leqq t \leqq T$, where $K_{1}=\left\{y: y \in K, \gamma_{0}(y) \leqq 1\right\}$. Since $K_{1}$ is weak $\Gamma$-compact, $f(t, y)$ is uniformly weakly $\Gamma$-continuous on $[0, T] \times K_{1}$, thus it follows, as in the proof of Theorem 4.1, that the sequence $y_{n}\left(t, \eta_{n}\right)$ satisfies the hypotheses of Lemma 3.1. Consequently that lemma implies that there exists a weak $\Gamma$-solution of (2.1) on $[0, T]$ such that $y_{T}(t) \in K_{1}$ on $[0, T]$ and $y_{T}(0) \in H$.

For each $n=1,2, \cdots$, let $y_{n}(t)$ be a weakly $\Gamma$-continuous function from $I$ to $K_{1}$ with $y_{n}(0) \in H$ and such that on $[0, n], y_{n}(t)$ is a weak $\Gamma$-solution of (2.1). The sequence $\left\{y_{n}(t)\right\}$ satisfies the hypotheses of Lemma 3.1, and the conclusion of the theorem follows by an application of that lemma.

Theorem 3 of [2] follows immediately from Theorem 4.1 above in view of the observation (ii) of [2], namely that the dual cone $K^{*}$ of a cone $K$ with interior has a weak* compact cross-section. If $\Gamma=Y^{*}$ then the solution of (2.1) whose existence is asserted in Theorem 4.1 is, by Lemma 2.2 and the remark following the statement of that lemma, a strong solution, in the extended sense, of (2.1) on $I$. If the mapping $f(t, y)$ of $I \times K$ to $-K$ is continuous with respect to the strong topology on $Y$ it is not difficult to see that a strong solution in the 
extended sense, of (2.1) is in fact a strong solution of (2.1). Thus Theorem 1 of [2] also follows from Theorem 5.1.

If $Y$ is finite dimensional, Theorem 5.1 implies a result similar to Theorem (*) of [4] referred to in $\S 1$. In the latter result the initial value $y(0)$ is asserted to lie on the surface of a sphere $\|y\|=C, C>0$, rather than on some hyperplane. For the finite dimensional case it is clear that the proof given above can be modified to give this different normalization of the initial value.

\section{REFERENCES}

1. N. Bourbaki, Topologic Générale, Chap. X, Actualities Scientifiques et Industrielles n. 1084, Paris 1949.

2. C. V. Coffman, Linear differential equations on cones in Bunach spaces, Pacific J. Math., 12 (1962), 69-75.

3. N. Dunford and B. J. Pettis, Linear operations on summable functions, Trans. Amer. Math. Soc., 47 (1940), 323-391.

4. P. Hartman and A. Wintner, On monotone solutions of non-linear differential equations, Amer. J. Math., 76 (1954), 860-886.

5. E. Hille and R. S. Phillips, Functional analysis and semi-groups, Amer. Math. Soc. Colloquium Publ. vol. XXXI, 2nd ed. 1957.

CaRnegie Institute of Technology 



\section{PACIFIC JOURNAL OF MATHEMATICS}

\section{EDITORS}

Robert Osserman

Stanford University

Stanford, California

M. G. Arsove

University of Washington

Seattle 5 , Washington
J. DugundjI

University of Southern Califorma: Los Angeles 7, California

Lowell J. Paige

University of California

Los Angeles 24, California

\section{ASSOCIATE EDITORS}

E. F. BECKENBACH

B. H. NeumanN

F. WOLF

K. YosIDA

\section{SUPPORTING INSTITUTIONS}

UNIVERSITY OF BRITISH COLUMBIA

CALIFORNIA INSTITUTE OF TECHNOLOGY

UNIVERSITY OF CALIFORNIA

MONTANA STATE UNIVERSITY

UNIVERSITY OF NEVADA

NEW MEXICO STATE UNIVERSITY

OREGON STATE UNIVERSITY

UNIVERSITY OF OREGON

OSAKA UNIVERSITY

UNIVERSITY OF SOUTHERN CALIFORNIA
STANFORD UNIVERSITY

UNIVERSITY OF TOKYO

UNIVERSITY OF UTAH

WASHINGTON STATE UNIVERSITY

UNIVERSITY OF WASHINGTON

AMERICAN MATHEMATICAL SOCIETY CALIFORNIA RESEARCH CORPORATION SPACE TECHNOLOGY LABORATORIES NAVAL ORDNANCE TEST STATION 


\section{Pacific Journal of Mathematics}

\section{Vol. 14, No. 1 \\ May, 1964}

Richard Arens, Normal form for a Pfaffian .........................

Charles Vernon Coffman, Non-linear differential equations on cones in Banach

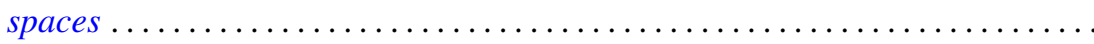

Ralph DeMarr, Order convergence in linear topological spaces ..............

Peter Larkin Duren, On the spectrum of a Toeplitz operator ................

Robert E. Edwards, Endomorphisms of function-spaces which leave stable all

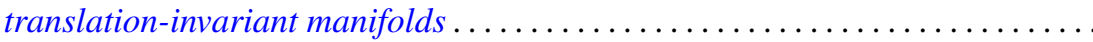

Erik Maurice Ellentuck, Infinite products of isols . . . . . . . . . . . . . . . . 49

William James Firey, Some applications of means of convex bodies . . . . . . . . 53

Haim Gaifman, Concerning measures on Boolean algebras ............. 61

Richard Carl Gilbert, Extremal spectral functions of a symmetric operator. . . . . . 75

Ronald Lewis Graham, On finite sums of reciprocals of distinct nth powers ..... 85

Hwa Suk Hahn, On the relative growth of differences of partition functions ...... 93

Isidore Isaac Hirschman, Jr., Extreme eigen values of Toeplitz forms associated

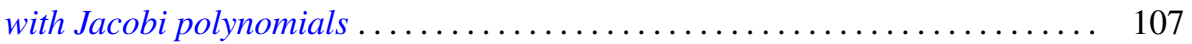

Chen-jung Hsu, Remarks on certain almost product spaces . . . . . . . . . . . 163

George Seth Innis, Jr., Some reproducing kernels for the unit disk . . . . . . . . . 177

Ronald Jacobowitz, Multiplicativity of the local Hilbert symbol . . . . . . . . . . . 187

Paul Joseph Kelly, On some mappings related to graphs ................. 191

William A. Kirk, On curvature of a metric space at a point . . . . . . . . . . . . 195

G. J. Kurowski, On the convergence of semi-discrete analytic functions . . . . . . . 199

Richard George Laatsch, Extensions of subadditive functions . . . . . . . . . . . 209

V. Marić, On some properties of solutions of $\Delta \psi+A\left(r^{2}\right) X \nabla \psi+C\left(r^{2}\right) \psi=0 \ldots 217$

William H. Mills, Polynomials with minimal value sets . . . . . . . . . . . 225

George James Minty, Jr., On the monotonicity of the gradient of a convex

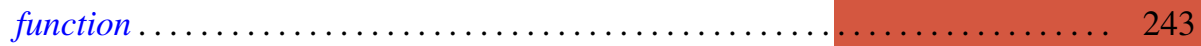

George James Minty, Jr., On the solvability of nonlinear functional equations of 'monotonic' type ................................... 249

J. B. Muskat, On the solvability of $x^{e} \equiv e(\bmod p) \ldots \ldots \ldots \ldots \ldots \ldots \ldots \ldots . \ldots \ldots$

Zeev Nehari, On an inequality of $P . R$. Bessack ................... 261

Raymond Moos Redheffer and Ernst Gabor Straus, Degenerate elliptic

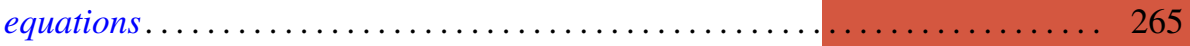

Abraham Robinson, On generalized limits and linear functionals . . . . . . . . . 269

Bernard W. Roos, On a class of singular second order differential equations with a

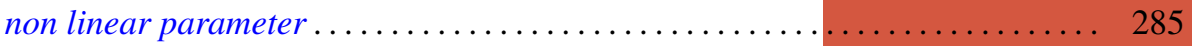

Tôru Saitô, Ordered completely regular semigroups . . . . . . . . . . . . . . . . 295

Edward Silverman, A problem of least area ....................... 309

Robert C. Sine, Spectral decomposition of a class of operators . . . . . . . . . 333

Jonathan Dean Swift, Chains and graphs of Ostrom planes . . . . . . . . . . . 353

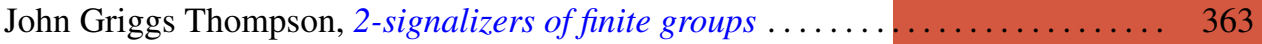

Harold Widom, On the spectrum of a Toeplitz operator . . . . . . . . . . . . . 365 